\title{
Day hospital treatment following brief inpatient medical stabilisation for adolescents with anorexia nervosa is equivalent in efficacy to inpatient treatment
}

\author{
Alexis Fertig, Jennifer Wildes \\ Department of Psychiatry, University of Pittsburgh, Pittsburgh, Pennsylvania, USA \\ Correspondence to Alexis Fertig; fertigam@upmc.edu
}

\section{WHAT IS ALREADY KNOWN ON THIS TOPIC?}

Aggressive treatment and weight restoration as early as possible offer the best chance of recovery from anorexia nervosa. ${ }^{1}$ Inpatient treatment is often used to ensure adequate weight restoration; however, there are significant drawbacks. In adolescents with anorexia nervosa, family involvement in the treatment is crucial to continued improvement and maintenance of recovery, ${ }^{2}$ but this can be difficult to attain in an inpatient setting. Furthermore, healthcare spending in most countries is not sustainable at current rates and it is vital to find ways to treat patients effectively while being respectful of limited resources. ${ }^{3}$

\section{WHAT DOES THIS PAPER ADD?}

- First study to provide a direct comparison of day treatment and inpatient programmes for adolescents with anorexia nervosa.

- Findings linked to clinical outcomes and financial burden on the healthcare system support the utility of day treatment programmes after medical stabilisation for anorexia nervosa in adolescents.

\section{LIMITATIONS}

- More than one-third of patients approached refused to participate. However, this is not unexpected in such a patient population, and no clear demographic differences were apparent between participating and non-participating groups. This study enrolled anorexia nervosa patients with no previous hospital admissions, which limits the generalisability of the results to more chronic patients.

\section{WHAT'S NEXT IN RESEARCH?}

It will be important to determine whether a more intensive investment in treatment early in the course of the illness mitigates the significant financial burden of anorexia nervosa. Another area of continued interest involves comparison of responders and non-responders to treatment, regardless of the level of care. Patients with more chronic anorexia nervosa have worse outcomes regardless of the treatment setting, and further research is needed to understand why this occurs.

\section{COULD THESE RESULTS CHANGE YOUR PRACTICES AND WHY?}

We are motivated to transition medically stable adolescents with anorexia nervosa to treatment in a day programme as early as possible. This study provides evidence in support of minimising the use of prolonged inpatient hospitalisation. This could expand the number of academic medical centre programmes with the resources to treat adolescents with anorexia nervosa. Nevertheless, treatment by providers with expertise in eating disorders is necessary regardless of the setting.

Competing interests None.

doi:10.1136/eb-2014-101890

\section{REFERENCES}

1. National Collaborating Centre for Mental Health (UK). Eating disorders: core interventions in the treatment and management of anorexia nervosa, bulimia nervosa and related eating disorders. Leicester, UK: British Psychological Society (UK), 2004.

2. Couturier J, Kimber M, Szatmari P. Efficacy of family-based treatment for adolescents with eating disorders: a systematic review and meta-analysis. Int J Eat Disord 2013;46:3-11.

3. Howard W, Evans K, Quintero-Howard C, et al. Predictors of success or failure of transition to day hospital treatment for inpatients with anorexia nervosa. Am J Psychiatry 1999;156:1697-702.

ABSTRACT FROM Herpertz-Dahlmann B, Schwarte R, Krei M, et al. Day-patient treatment after short inpatient care versus continued inpatient treatment in adolescents with anorexia nervosa (ANDI): a multicentre, randomised, open-label, non-inferiority trial. Lancet 2014;383:1222-9.

Patients/participants One hundred and seventy-two females aged 11-18 during their first admission to hospital for anorexia nervosa DSM IV.

Setting Five university hospitals and one major general hospital for child and adolescent psychiatry in Germany; recruitment February 2007 to April 2010. Intervention Day patient care after brief inpatient stabilisation $(n=87)$. Participants were admitted to hospital for 3 weeks, followed by treatment as a day patient from 8.00 to 16.30 on weekdays. The programme included weight restoration, nutritional counselling, cognitive behavioural therapy and family therapy. Hospital discharge occurred when the target weight had been reached for 2 weeks. Participants were then assigned to an outpatient treatment programme.

Comparison Continued inpatient care $(n=85)$. Participants followed the same multidisciplinary treatment programme and discharge criteria as the intervention group, but they received all care prior to discharge as an inpatient. Patient follow-up Seventy-one per cent of the intervention group and $88 \%$ of the control group completed treatment; $99 \%$ of the intervention group (1 lost to follow-up) and 88\% of the control group (10 lost to follow-up) were included in modified intention-to-treat analysis.

Allocation: Concealed; randomisation stratified within sites to balance for age and BMI.
Blinding: Single blind (assessors)

\section{OUTCOMES}

BMI at 12 months or at readmission Day care was not inferior to continued inpatient care (mean difference $=0.46,95 \%$ CI-0.11 to 1.02 ).

Morgan and Russell Average Outcome Score (MRAOS) There was no difference in total MRAOS score between the two groups (score difference $=0.64,95 \%$ CI-0.05 to 1.34 ).

Number of readmissions $15.1 \%$ of the day care group were readmitted, compared to $25.3 \%$ of the inpatient group (difference $=-10.2 \%, 95 \% \mathrm{CI}$ -22.7 to 2.2 ).

Continued diagnosis of anorexia nervosa $23 \%$ of the day care group and $24 \%$ of the inpatient group still fulfilled diagnostic criteria at 12 months. Adverse events There was no difference in the frequency of treatment-related adverse events (seven in the day care group vs eight in the inpatient group). Suicidal ideation occurred in two females in the day care group and three in the inpatient group. A suicide attempt was made in the day care group 3 months after discharge. 